\title{
Effectiveness of Platelet Rich Fibrin (PRF) Compared with Conventional Therapy Toward Wound Dehiscence of Post-Operative Laparotomy
}

\section{Abdurahman Laqif, Nur Aini Fatah, and Supriyadi Hari Respati}

Department of Obstetrics and Gynecology Faculty of Medicine of Universitas Sebelas Maret/ Dr. Moewardi General Hospital Surakarta

\section{Abstract}

Objective: To compare the effectiveness of Platelet Rich Fibrin (PRF) and conventional therapy toward wound dehiscence of post-operative laparotomy in the Department of Obstetrics and Gynecology at Moewardi hospital.

Methods: This was a cross-sectional study conducted from September to December 2017 on obstetrics and gynecology post-laparotomy patients undergoing subtotal wound dehiscence in the Department of Obstetrics and Gynecology at Moewardi

Corresponding Author:

Abdurahman Laqif

a_laqif@yahoo.com

Received: 23 February 2019

Accepted: 6 March 2019

Published: 25 March 2019

Publishing services provided by Knowledge E

(c) Abdurahman Laqif et al. This article is distributed under the terms of the Creative Commons Attribution License, which permits unrestricted use and redistribution provided that the original author and source are credited.

Selection and Peer-review under the responsibility of the ICO-HELICS Conference Committee. hospital from 2013 to 2017. They were divided into 2 groups, group 1 consisted of patients with subtotal wound dehiscence after obstetric and gynecological operation receiving conventional non-operative therapy, and group 2 was those having subtotal wound dehiscence after obstetrics and the gynecological operation receiving non-operative treatment with PRF. The data were analyzed using the T-test, performed by SPSS (Software Package for Social Science).

Results: There were 15 subjects in each group meeting the inclusion and exclusion criteria for both case and control groups. The statistical test showed that the average duration of wound healing in control group (12.60 about 2.58) was longer than in PRF group (7.0 about 3.52) with the significance of value $p=0.001(p<0.05)$. The PRF group spent less cost (IDR 4.511.362 $\pm 2.977 .934)$ compared with the conventional therapy group (IDR 12.540.735 $\pm 8.227 .433)$ with $p=0.001(p<0.05)$.

Conclusions: Patients with PRF therapy had faster-wound healing duration, and the result was statistically significant with $p=0.001(p<0.05)$. PRF therapy also had more cost-effectiveness compared with conventional therapy with $p=0.001(p<0.05)$.

Keywords: Wound healing, Platelet-rich fibrin

\section{Background}

Wound dehiscence after laparotomy remains a severe surgical complication problem and includes $1-3 \%$ of patients undergoing abdominal surgery. The occurrence of postlaparotomy wound dehiscence is related to conditions such as anemia, hypoalbuminemia, malnutrition, malignancy, obesity, diabetes, geriatrics, as well as specific surgical 
procedures such as obstetric and gynecological surgery (obstetrical and gynecological laparotomy) [1]. Current dehiscence management is divided into non-operative or conservative and operative management. Platelet Rich Fibrin (PRF) is a breakthrough for non-operative therapy in patients with surgical dehiscence. PRF contains many growth factors needed for wound healing [2]. PRF contains over 30 bioactive proteins, growth factors, various cytokines, and chemokines that play an essential role in wound healing, acute tissue response to trauma, and involves in some of the physiological processes of the cell, such as growth differentiation, and cell replication [3]. PRF is autologous, so it is relatively safe and does not pose a risk of allergic or infectious disease. This study aimed to determine the effectiveness of wound healing in wound dehiscence of obstetric and gynecologic laparotomy with PRF treatment compared to conventional non-operative methods.

\section{Subject and Methods}

This was a cross-sectional study conducted in patients who admitted to Obstetric and Gynecology clinic of Dr. Moewardi General Hospital from September to December 2017 and underwent subtotal wound dehiscence following obstetric and gynecological laparotomy in the last 5 years. Subjects were divided into two groups: the control group was given conventional non-operative method while the treatment group was using PRF treatment and all the subjects met inclusion and exclusion criteria. Inclusion criteria including post-obstetric and gynecologic laparotomy dehiscence with a minimum wound length of $3 \mathrm{~cm}$ with wound depth up to the fascia of musculus rectus abdominis, while exclusion criteria including post-obstetric and gynecologic, obstetric deformation complications with depth to peritoneum parietal, complications of laparoscopic dehiscence wounds were present such as infection, worsening of the patient's general condition, and also the patient's refusal to continue the study. The sample size was taken with fixed disease sampling method, and Patient has first assessed the condition of the wound then divided into two groups. In the control group using conventional methods, wound dressing was performed by cleaning the wound with $0.9 \%$ sterile saline $(\mathrm{NaCl})$ followed by wound closure using sterile gauze. In the PRF therapy group, wound dressing was performed by cleaning the wound with $0.9 \%$ sterile saline $(\mathrm{NaCl})$ followed by PRF gel and then wound closure by sterile gauze. Evaluation of wound conditions were 3 days after treatment for each group, followed by the retrieval and data processing and conducted statistical analysis. The difference among 2 groups was considered statistically significant if $p$ value $<0.05$. 


\section{Results}

The subjects of the study were patients with subtotal wound dehiscence after obstetric and gynecologic laparotomy in Dr. Moewardi Hospital. Subjects were divided into 2 groups, each group consisted of 15 patients. Each group's characteristics is shown in Table 1 below:

TABLE 1: Description of Data Subject of the Study.

\begin{tabular}{|c|c|c|c|c|c|}
\hline Variable & $\mathrm{N}$ & Min & Max & Mean & SD \\
\hline \multicolumn{6}{|c|}{ Maternal Age (Year) } \\
\hline Control group & 15 & 26,00 & 43,00 & 36.06 & 5.22 \\
\hline PRF group & 15 & 25,00 & 43,00 & 35.38 & 5.02 \\
\hline \multicolumn{6}{|l|}{ BMI } \\
\hline Control group & 15 & 20,00 & 36,50 & 22,36 & 4,13 \\
\hline PRF group & 15 & 19,00 & 24,00 & 21,13 & 1,46 \\
\hline \multicolumn{6}{|l|}{ Hemoglobin } \\
\hline Control group & 15 & 9,30 & 12,00 & 11,47 & 3,03 \\
\hline PRF group & 15 & 9,30 & 11,90 & 10,51 & 0,75 \\
\hline \multicolumn{6}{|l|}{ Albumin } \\
\hline Control group & 15 & 2,50 & 4,00 & 3,37 & 0,37 \\
\hline PRF group & 15 & 3,00 & 4,10 & 3.56 & 0,35 \\
\hline \multicolumn{6}{|l|}{ Blood Glucose } \\
\hline Control group & 15 & 80,00 & 112,00 & 95,00 & 10,336 \\
\hline PRF group & 15 & 68,00 & 120,00 & 95,00 & 13,824 \\
\hline Working status & & Work & \multicolumn{3}{|c|}{ Do Not Work } \\
\hline Control group & 15 & 7 & & 8 & \\
\hline PRF group & 15 & 6 & & 9 & \\
\hline
\end{tabular}

Statistical analysis was done to determine the normality and the homogeneity of the data, continued with bivariate analysis of each characteristic above. We found that all the above variables were homogeneous and there were no significant differences.

The outcome of wound healing between the control group and PRF is seen in the average time of wound healing as shown in Table 2 below:

TABLE 2: Wound healing time between the control group and PRF group.

\begin{tabular}{l|c|c|c|c|}
\hline Variable & Group & N & Mean (days) & $p$ \\
\hline Wound healing time & Control group & 15 & $12.60 \pm 2.58$ & $0.001^{*}$ \\
\hline & PRF group & 15 & $7.0 \pm 3.52$ & \\
* Significance value $\mathrm{p}<0.05$ & &
\end{tabular}

Table 2 shows that the average length of wound healing in the control group was longer than PRF group $(12.60 \pm 2.58$ vs $7.0 \pm 3.52)$. Statistical analysis using the normality 
data test by Saphiro-Wilk test on the duration of wound healing for the control group and the PRF group showed $p=0.050$ and $p=0.069$ ( $p$ sig $>0.05$ ), which means that data is normally distributed. The homogeneity test of Lavene showed $p=0.23$ ( $p$ sig $>0.05$ ), meaning that the data is homogeneous. The statistical difference in wound healing time in both groups then analyzed using unpaired t-test. It showed that there was a very significant difference $(p=0.001)$ between two groups.

TABLE 3: Multivariate Dependent Regression independent Age, BMI, HB, Albumin, Blood Glucose Serum (BGs), and PRF toward dependent variable (Wound Healing Time).

\begin{tabular}{l|c|c|c|c|}
\hline Variable & $\begin{array}{c}\text { Standardized } \\
\text { Coefficient }(\beta)\end{array}$ & $T$ & $p$ & Note \\
\hline Umur & 10,169 & $-0,064$ & 0,950 & Not significant \\
\hline Body mass index (BMI) & $-0,010$ & 0,274 & 0,786 & Not significant \\
\hline Hemoglobin (Hb) & 0,058 & 1,461 & 0,157 & Not significant \\
\hline Albumin & 0,462 & 0,102 & 0,920 & Variable \\
\hline Blood glucose & 0,181 & 0,122 & 0,904 & Variabele \\
\hline PRF & 0,008 & $-3,699$ & 0,001 & Signifiant \\
\hline Value of $\mathrm{F}=4,367$ p Sig $=\mathbf{0 , 0 0 4}$ (Significant) & & \\
\hline
\end{tabular}

F test on double linear regression which aimed to test independent variable (age, $\mathrm{BMI}, \mathrm{Hb}$, albumin, blood glucose, PRF) on regression model shows that all independent variables had a simultaneous effect on the dependent variable (wound healing time).

The costs of therapy on patients undergoing wound dehiscence after obstetric and gynecologic laparotomy in Dr. Moewardi Hospital over the past five years (2013-2017) were also analyzed in both control group and PRF group, the cost data obtained from the medical record, with an average cost as follows:

TABLE 4: Average Cost of therapy on the control group and PRF group.

Variable
The average cost of
therapy
* Significance value $p<0.05$

\begin{tabular}{c|c|c|}
\hline Group & N & Average cost (days) \\
\hline Conventional & 15 & IDR 12.540.735 \pm 8.227 .433 \\
\hline PRF & 15 & IDR 4.511.362 \pm 2.977 .934 \\
\hline
\end{tabular}

From Table 3 it was found that the average cost of therapy in conventional group patients was more expensive than the PRF group (12.540.735 $\pm 8.227 .433 \mathrm{v}$ s 4.511.362 $\pm 2.977 .934)$ and it is statistically significant ( $p=0.001)$. Thus PRF therapy can be used as a cheaper therapy.

Multivariate test by using confidence interval $95 \%$ and $\alpha=0.05$ showed that total wound healing cost between conventional and PRF group was not affected by external variables where $p=0.071(p<0,05)$. 
TABLE 5: A multivariate test of wound healing average cost between conventional and control group.

\begin{tabular}{|l|c|c|c|c|}
\hline Variables & \multicolumn{3}{|c|}{ Sexual Disorder } \\
\hline Occupational status & OR & \multicolumn{2}{|c|}{ Cl $95 \%$} & $\mathrm{P}$ \\
\hline Age & .785 & -4362335.260 & 9647326.682 & .442 \\
\hline Wound healing time & .944 & -345836.946 & 920818.425 & .356 \\
\hline Hb & .349 & -718962.486 & 1008476.604 & .731 \\
\hline BMl & -.265 & -1542027.120 & 1193814.280 & .794 \\
\hline Albumin & 1.408 & -293029.694 & 1522950.983 & .174 \\
\hline Blood Glucose serum & .294 & -6305767.124 & 8383344.365 & .772 \\
\hline Group & .202 & -257044.011 & 312501.243 & .841 \\
\hline * Significance $\mathrm{p}<0.05$ & -1.809 & -15944929.811 & 1107558.516 & .085 \\
\hline
\end{tabular}

\section{Discussion}

From the result of the bivariate test conducted on the research variables in both groups of PRF, it showed that age, BMI, hemoglobin, albumin, and blood glucose are not statistically different (or homogeneous) with $p>0.05$. The average duration of wound healing appears to be longer in the conventional group (12.60 \pm 2.58 days), compared to PRF group (7.00 \pm 3.52 days). The unpaired t-test analysis using a 95\% confidence interval and $\alpha=0.05$ proved that the difference in wound healing among the conventional group and the PRF group was statistically significant $(p=0.001)$.

The Mann Whitney's test analysis using 95\% confidence interval and $\alpha=0.05$ proved that there was a very significant difference $(p=0.001)$ in total cost of wound healing between the conventional group and PRF group. Multivariate test using 95\% confidence interval and $\alpha=0.05$ showed that the existing external variable did not affect total wound healing cost between conventional group and PRF group ( $p=0.071 ; p>0.05)$.

Plasma Rich Fibrinogen is a blood plasma contain rich of fibrin and thrombocyte concentration 3-5 times above normal value (platelet concentration in whole blood) [4]. Another literature mentioned that thrombocyte concentrations in PRF were 2-8 times higher compared with average values [5]. The high concentration of platelets and various growth factors in PRF has made this agent applicable in many branches of medicine, i.e., oral surgery, plastic surgery, craniofacial surgery, cardiac surgery, orthopedics, neurology, sports medicine, and dermatology [6].

Plasma Rich Fibrinogen has various growth factors that are needed in wound healing. When platelets in PRF are activated by thrombin, thrombocytes release growth factors 
and other substances that serve to accelerate wound healing by increasing cell proliferation, matrix formation, osteoid production, tissue healing, angiogenesis, and collagen synthesis. Plasma Rich Fibrinogen works by degranulation of $\alpha$-granules present in platelets containing growth factors [7].

These growth factors help wound healing by attracting cells in a newly formed matrix and trigger cell division. Plasma Rich Fibrinogen has several advantages such as simple, safe, low cost, and clinical effectiveness so that PRF can be an ideal therapy for the treatment of chronic wounds [8]. Plasma Rich Fibrinogen not only releases growth factors in high concentrations but also has antimicrobial properties that can prevent infection because it contains leukocytes [9].

Plasma Rich Fibrinogen has long been used in healing wounds and soft tissue underneath. The use of PRF in the field of skin surgery and cosmetic dermatology is also proliferating [10]. Wound healing is a complex event involving various cells, and biochemical mediators, one of the critical part were platelets. In response to tissue damage, activated platelets will form platelet plugs and release various growth factors [11]. These growth factors play an essential role in the various phases of wound healing (the inflammatory phase up to the remodeling phase) [12]. Platelets also store other bioactive proteins, such as the enzyme matrix metalloproteinase (MMP), coagulation factors, chemotactic factors, adhesion molecules, vasoactive substances, as well as some bactericidal and fungicidal proteins that also play an active role in the wound healing process. Invitro, there is a relationship between platelet concentration with mesenchymal stem cell proliferation, fibroblast proliferation, and type I collagen production. It supports the role of PRF to accelerate wound healing [13].

Platelet Derived Growth Factor (PDGF) functions in the stimulation of fibroblasts, chemotactic, TGF- $\beta$ stimulation, collagen production, increased proteoglycan synthesis. Transforming Growth Factor Beta (TGF- $\beta$ ) functions to modulate the proliferation of fibroblasts, formation of extracellular matrix, increase collagen production by fibroblasts, neutrophil chemotactic factors, and macrophages, while Basic Fibroblast Growth Factor (bFGF) plays a role in collagen production, stimulation of angiogenesis, and myoblast proliferation [11].

In this study, besides healing wounds more quickly, PRF also has more affordable cost therapy than conventional therapy. Faster healing time affects hospitalization care time in the hospital, thus reducing cost therapy. Thus, PRF can be a practical and cost-effective treatment option. 


\section{Conclusion}

There was a significant difference in wound healing time of wound dehiscence patients after obstetric and gynecological laparotomy using PRF therapy compared to conventional methods, where PRF group has shorter wound healing time than the control group. Also, PRF therapy is cheaper than conventional therapy.

\section{Recommendation}

Further study is needed by involving more subjects so it will strengthen the validity and the study could be used as a comparison.

\section{References}

[1] Ramneesh, G., Sheerin, S., Surinder, S., \& Bir, S. A prospective study of predictors for post laparotomy abdominal wound dehiscence. Journal of Clinical and Diagnostic Research: JCDR, 2014: 8(1), 80-3.

[2] Alavi, S., Malek, F., Eshghi, P, Arzhangian, H. Autologous platelet-rich plasma for the healing of an oncologic dehisced wound. APSP Journal of Case Reports, 2013: 4(3), 44.

[3] Schär, M. O, Diaz-Romero, J, Kohl, S., Zumstein, M. A., \& Nesic, D. Platelet-rich concentrates differentially release growth factors and induce cell migration in vitro, 2015: 473(5).

[4] Jadhav, G. R., Shah, D., \& Raghvendra, S. S. (2015). Autologous Platelet Rich Fibrin aided Revascularization of an immature, non-vital permanent tooth with apical periodontitis: A case report, 2015: 6(1).

[5] Pathak, H., Mohanty, S, Urs, A. B, Dabas, J. Treatment of Oral Mucosal Lesions by Scalpel Excision and Platelet-Rich Fibrin Membrane Grafting: A Review of 26 Sites. Journal of Oral and Maxillofacial Surgery, 2015; 73(9), 1865-1874.

[6] Kawase, T., Kamiya, M., Kobayashi, M., Tanaka, T., Okuda, K., Wolff, L. F., \& Yoshie, H. The heat-compression technique for the conversion of platelet-rich fibrin preparation to a barrier membrane with a reduced rate of biodegradation. Journal of Biomedical Materials Research. Part B, Applied Biomaterials, 2015; 103(4), 825-31.

[7] Sun, X.-L., Zhou, Y.-M., Zhao, J.-H., Zheng, L., \& Yang, T. The effect of plateletrich fibrin on biologic characteristics of osteoblasts]. Shanghai Kou Qiang Yi Xue Shanghai Journal of Stomatology, 2015; 24(1), 61-4. 
[8] Oliveira, M R., de Silva, A., Ferreira, S., Avelino, C, Garcia, I R, Mariano, R.. (2015). Influence of the association between platelet-rich fibrin and bovine bone on bone regeneration. A histomorphometric study in the calvaria of rats., 2015; 44(5).

[9] Hehn, J., Schwenk, T., Striegel, M., \& Schlee, M. The effect of PRF (platelet-rich fibrin) inserted with a split-flap technique on soft tissue thickening and first marginal bone loss around implants: results of a randomized, controlled clinical trial. International Journal of Implant Dentistry, 2016; 2(1), 13.

[10] Hatakeyama, I., Marukawa, E., Takahashi, Y., \& Omura, K. Effects of platelet-poor plasma, platelet-rich plasma, and platelet-rich fibrin on the healing of extraction sockets with buccal dehiscence in dogs. Tissue Engineering. Part A, 2014; 20(3-4), 874-82.

[11] Jayadev, M., Marshal, Vr., Naik, Karunakar, P. Role of Platelet-rich fibrin in wound healing: A critical review. Journal of Conservative Dentistry, 2013; 16(4), 284.

[12] Fresno L, Fondevila, D., Bambo O, Chacaltana, A., García, F, Andaluz, A. Effects of platelet-rich plasma on intestinal wound healing in pigs. The Veterinary Journal, 2010; 185(3), 322-327.

[13] Doiphode AM, Hegde P, Mahindra U, Santhosh Kumar SM, Tendulkar PD, Tripathi V. Evaluation of the efficacy of platelet-rich plasma and platelet-rich fibrin in alveolar defects after removal of impacted bilateral mandibular third molars. Journal of International Society of Preventive \& Community Dentistry, 2016; 6(Suppl 1), S47-52. 